\title{
TB or not TB? Improving the understanding and diagnosis of tuberculosis through metabolomics
}

\author{
"...metabolomics has resulted in an exponential increase in the \\ number of newly identified tuberculosis biomarkers, which has not \\ only shed light on previously unknown disease mechanisms, but could \\ potentially contribute to all aspects of tuberculosis clinical care..."
}

First draft submitted: 27 July 2016; Accepted for publication: 12 August 2016; Published online: 19 September 2016

Keywords: biomarkers $\bullet$ diagnostics $\bullet$ metabolite markers $\bullet$ metabolomics $\bullet$ Mycobacterium tuberculosis • tuberculosis

Despite the major discovery in 1882 by Robert Koch that the causative agent for tuberculosis (TB) is the infectious bacteria Mycobacterium tuberculosis, and all the genomics, transcriptomics and proteomics data collected on this organism, and the subsequent vaccination, diagnostic and treatment approaches developed since then, TB is still a major global health problem. TB reportedly occurs in approximately a third (1.9 billion) of the world's population either in its active (symptomatic) or latent (asymptomatic) form, which subsequently results in the death of 1.5 million individuals per annum, ranking it the world's foremost cause of death from a single infectious bacterial agent [1].

Smear microscopy is currently the most commonly used method for diagnosing active TB in third-world countries. Despite the fact that it is a low-cost, quick and simple method, it has a sensitivity of only $62 \%$; and it cannot distinguish between various Mycobacterium species; nor can it detect drug resistance [2]. The current gold standard for diagnosing TB, however, functions on the basis of bacterial culturing, and typically takes about 2 weeks at best for a result to be obtained [3]. In that period, an individual with active TB could infect 10-15 individuals, depending on his/her social habits. Apart from this serious draw- back, sophisticated laboratory infrastructure and highly trained staff are required, which are not necessarily available in many thirdworld communities where the prevalence of $\mathrm{TB}$ is at its highest. There are currently other newer TB-diagnostic technologies available which are faster, such as nucleic acid amplification methods [4], phage assays [5] and serological tests [6]. Unfortunately, however, these also fall short with regard to their high cost and limited sensitivities, subsequently missing many TB-positive cases and contributing to the estimated 2.9 million missed TB cases globally each year [1]. Another drawback in current TB-diagnostic methods is that sputum is obtained with difficulty in childhood TB and HIV co-infected cases, subsequently contributing to the aforementioned missed cases. TB-diagnostic methods using urine or blood would be considered less invasive and easier to collect from a patient (when one considered sputum collection in children often requires gastric aspirate) and could potentially pick up patient groups that current diagnostic technologies miss. Considering this, the world is in desperate need for new TB-diagnostic approaches which are fast, inexpensive, sensitive, selective, easy to operate without extensive training or specialized skills and portable (point-of-care devices). They should also be able to identify

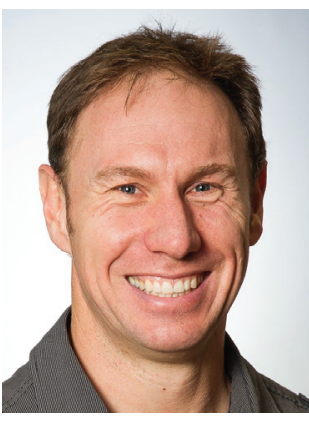

Du Toit Loots

Human Metabolomics, NorthWest University, Hoffman Street, Private Bag X6001, Box 269, Potchefstroom 2531, South Africa Tel.: +27182991818

dutoit.loots@nwu.ac.za 
or differentiate between various Mycobacterium species, determine their sensitivity or resistance to medication and predict or monitor first-line treatment outcome well before the 6 -month treatment period is completed (perhaps even before treatment commences).

\section{"...metabolomics has allowed for better} understanding of the adaptations of Mycobacterium tuberculosis to the host's defense and vice versa..."

In an attempt to improve these strategies, some research groups have opted to address the problem by looking at it from a different perspective, using one of the newest omics technologies available, termed metabolomics, in order to identify new TB biomarkers. These new markers can subsequently be used for better understanding of the disease and the host's response to it, and subsequently the development of improved diagnostic and treatment strategies. Metabolomics is broadly defined as the nonbiased identification and quantification of the metabolites (or small molecular weight chemical compounds) present in a biological system or a sample, using an assortment of highly specialized analytical techniques, in combination with various computational, statistical and mathematical analyses [7]. Since the metabolome is the ultimate downstream product of the genome, transcriptome and proteome, a disturbance in any of these, due to any inherited or acquired perturbation, will alter the host metabolome with regard to the presence or absence of specific metabolites and/or their quantities. Additionally, the direct presence of a pathogen in these samples, or the host's response to the pathogen, would also modify the metabolome. Subsequently, in the context of TB, a number of metabolomics studies have been carried out to date, searching for new markers using patient collected sputum $[8,9]$. Because of the fact that these altered metabolite markers or metabolite patterns are not only due to the presence of the foreign body in the host, but also due to a specific host response, patient samples carrying little/none of the infectious organism, such as blood [10,11] or urine [12], can also be used for new biomarker identification. Using serum markers obtained via an LC-MS-based metabolomics approach, Feng et al. [10] obtained an area under the receiver operator curve (area under the curve) value of 0.991, and Che et al. [11], an area under the curve value of 0.85 , when using a GC-MS-based metabolomics approach. The advantages of using these samples over that of sputum are that they are more easily attainable and require less invasive collection procedures, in addition to offering a lower risk of infection during handling and transport. At this point, it is also important to mention how metabolomics has contributed to new bio- marker identification for the purpose of TB diagnostics, using patient-collected breath, probably one of the least invasive sample collection approaches for diagnosing this disease to date. Using GC-MS-identified volatile organic compound profiles, Phillips et al. [13] were able to identify TB with a sensitivity of $84 \%$ and a specificity of $64.7 \%$, and Kolk et al. [14] with a sensitivity of $62 \%$, a specificity of $84 \%$ and an accuracy of $77 \%$. Furthermore, metabolomics has been shown to be a valid approach for identifying markers characterizing/identifying various infectious Mycobacterium species with probabilities ranging from 72 to $100 \%$, depending on the species of Mycobacterium, using as little as $1 \times 10^{3}$ cells [15], as well as drug-resistant strains [16,17]. Considering this, metabolomics is proving to be a valuable tool for new TB-diagnostic biomarker detection, considering the need for such a method to not only detect TB, but to also differentiate between various infectious Mycobacterium species and drug-resistant strains, in a highly sensitive and selective manner. Ideally, these techniques could also predict treatment outcome using patient samples collected via less intrusive methods, with a lower risk of infection to the clinical staff. The costs of the highly specialized analytical techniques required for detecting these markers, and the specialized skills required to operate these apparatus, may be seen as a drawback. The general idea, however, is that these expensive, selective and highly specialized apparatus would only be used in the biomarker identification phase in the context of a research environment, to aid in the identification and development of simpler detection methods specific to the identified diagnostic biomarkers. These could then be applied in the clinical context, for example, using nanotechnology-based detection approaches. This will subsequently allow for the use of these diagnostic biomarkers in the form of a rapid, inexpensive, sensitive and easy-to-use detection device, which does not require extensive training or sophisticated infrastructure, and can subsequently be used for point-of-care/bedside TB diagnostics. Alternatively, this approach can also be used toward high-throughput laboratory-based methods, where hundreds of samples are run simultaneously with minimal preparation, using inexpensive equipment such as a basic spectrophotometer, for instance.

Apart from their diagnostic applications, various newly identified TB biomarkers have additionally contributed to the existing knowledge of the biology of the causative pathogen [16], as well as to various underlying disease mechanisms [9]. These include mechanisms related to $M$. tuberculosis drug resistance $[16,17]$ and virulence [18] including upregulation of various antioxidant pathways, the use of alkanes and fatty acids as alternative energy substrates $[16,17,19]$, a shift in aconitase functionality toward mRNA binding and stability [17] and cell 
wall remodeling [17,18]). Anti-TB drug mechanisms [20] and side effects in the host have also been elucidated including anti-TB drug inhibition of the host's electron transport chain resulting in oxidative stress and inducing an abnormal organic acid profile [21]. What is of even greater interest, currently, is that metabolomics has allowed for better understanding of the adaptations of $M$. tuberculosis to the host's defense and vice versa, and subsequently the discovery of the presence of a citramalate cycle in $M$. tuberculosis, and the interaction of this cycle with an upregulated glyoxylate cycle during infection; an increased utilization of fatty acids and glutamate by $M$. tuberculosis during infection; an additional mechanism by which the host manufactures hydrogen peroxide in order to eliminate the infecting bacteria; inhibition of the electron transport chain of the host by $M$. tuberculosis, resulting in elevation in various neurotransmitters and organic acids, better explaining the occurrence of various TB-associated symptoms [9]; and clues to improved treatment approaches [12]. Considering this, metabolomics has shed light on never before identified metabolic pathways in both man and the TBcausing bacteria, which in time will undoubtedly contribute to improved treatment strategies, and ultimately assist in curbing the epidemic.

\section{References}

1 World Health Organization. Tuberculosis: WHO Global Tuberculosis Report 2014. World Health Organization, Geneva, Switzerland, 2 (2014).

2 Dhingra V, Aggarwal N, Rajpal S, Aggarwal J, Gaur S. Validity and reliability of sputum smear examination as diagnostic and screening test for tuberculosis. Indian J. Allergy Asthma Immunol. 17(2), 67-69 (2003).

3 Moore DF, Curry JI. Detection and identification of Mycobacterium tuberculosis directly from sputum sediments by Amplicor PCR. J. Clin. Microbiol. 33(10), 2686-2691 (1995).

4 Kim JH, Kim YJ, Ki CS, Kim JY, Lee NY. Evaluation of Cobas TaqMan MTB PCR for detection of Mycobacterium tuberculosis. J. Clin. Microbiol. 49(1), 173-176 (2011).

5 Albert H, Heydenrych A, Brookes R et al. Performance of a rapid phage-based test, FASTPlaqueTB, to diagnose pulmonary tuberculosis from sputum specimens in South Africa. Int. J. Tuberc. Lung Dis. 6(6), 529-537 (2002).

6 Lee J, Yu F, Lin M et al. Utility of immunochromatographic assay for detecting Mycobacterium tuberculosis from positive BACTEC MGIT 960 cultures. J. Biomed. Lab Sci. 22(64), e) (2010).

7 Dunn WB, Bailey NJC, Johnson HE. Measuring the metabolome: current analytical technologies. Analyst 130(5), 606-625 (2005).

8 Schoeman JC, Du Preez I, Loots DT. A comparison of four sputum pre-extraction preparation methods for identifying
Over the past 8 years, metabolomics has resulted in an exponential increase in the number of newly identified TB biomarkers, which has not only shed light on previously unknown disease mechanisms, but could potentially contribute to all aspects of TB clinical care, especially to that of improved TB diagnostics. Furthermore, metabolomics shows the capacity for identifying markers predicting treatment outcome, and could subsequently accelerate the time-consuming process of new anti-TB medication efficacy testing, and elucidating their mechanisms of action. Considering this, in the future, it would be beneficial to see increased efforts toward validating these newly identified biomarkers for use in a clinical context, especially toward improved diagnostics and anti-TB treatment strategies.

\section{Financial \& competing interests disclosure}

The author has no relevant affiliations or financial involvement with any organization or entity with a financial interest in or financial conflict with the subject matter or materials discussed in the manuscript. This includes employment, consultancies, honoraria, stock ownership or options, expert testimony, grants or patents received or pending, or royalties.

No writing assistance was utilized in the production of this manuscript.

and characterising $M$. tuberculosis using GCxGC-TOFMS metabolomics. J. Microbiol. Methods 91, 301-311 (2012).

9 Du Preez I, Loots DT. New sputum metabolite markers implicating adaptations of the host to Mycobacterium tuberculosis, and vice versa. Tuberculosis 93(3), 330-337 (2013).

10 Feng S, Du YQ, Zhang L, Zhang L, Feng RR, Liu SY. Analysis of serum metabolic profile by ultra-performance liquid chromatography-mass spectrometry for biomarkers discovery: application in a pilot study to discriminate patients with tuberculosis. Chin. Med. J. 128(2), 159-168 (2015).

11 Che N, Cheng J, Li H et al. Decreased serum 5-oxoproline in TB patients is associated with pathological damage of the lung. Clin. Chim. Acta 423, 5-9 (2013).

12 Luies L, Loots DT. Tuberculosis metabolomics reveals adaptations of man and microbe in order to outcompete and survive. Metabolomics 12(40), 1-9 (2016).

13 Phillips M, Basa-Dalay V, Bothamley G et al. Breath biomarkers of active pulmonary tuberculosis. Tuberculosis 90(2), 145-151 (2010).

14 Kolk AHJ, van Berkel JJBN, Claassens MM et al. Breath analysis as a potential diagnostic tool for tuberculosis. Int. J. Tuberc. Lung Dis. 16(6), 777-782 (2012).

15 Olivier I, Loots DT. A metabolomics approach to characterise and identify various Mycobacterium species. J. Microbiol. Methods 88(3), 419-426 (2012).

16 Loots DT. An altered Mycobacterium tuberculosis metabolome induced by kat $G$ mutations resulting in isoniazid resistance. Antimicrob. Agents Chemother. 58(4), 2144-2149 (2014). 
17 Loots DT. New insights into the survival mechanisms of rifampicin resistant tuberculosis. J. Antimicrob. Chemother 71, 655-660 (2016).

18 Meissner-Roloff RJ, Koekemoer G, Warren RM. A metabolomics investigation of a hyper- and hypo-virulent phenotype of Beijing lineage M. tuberculosis. Metabolomics 8(6), 1194-1203 (2012).

19 Rhee KY, De Carvalho LP, Bryk R et al. Central carbon metabolism in Mycobacterium tuberculosis: an unexpected frontier. Trends Microbiol. 19(7), 307-314 (2011).
20 Halouska S, Fenton RJ, Barletta RG, Powers R. Predicting the in vivo mechanism of action for drug leads using NMR metabolomics. ACS Chem. Biol. 7(1), 166-171 (2012).

21 Loots DT, Wiid IsJ, Page BJ, Mienie LJ, Helden PD. Melatonin prevents the free radical and MADD metabolic profiles induced by antituberculosis drugs in an animal model. J. Pineal Res. 38(2), 100-106 (2004). 